\title{
Shapes and Fall Orientations of Ice Particle Aggregates
}

\author{
Zhiyuan Jiang, Johannes Verlinde, and Eugene E. ClothiauX \\ Department of Meteorology and Atmospheric Science, The Pennsylvania State University, University Park, Pennsylvania \\ KULTEGIN AYdin \\ Department of Electrical Engineering, The Pennsylvania State University, University Park, Pennsylvania
}

CARL SCHMITT

National Center for Atmospheric Research, Boulder, Colorado

(Manuscript received 21 August 2018, in final form 22 March 2019)

\begin{abstract}
Testing the often-made assumption that ice particle aggregates (snowflakes) are well represented by oblate spheroids, ellipsoid fits are applied to aggregate images. An algorithm to retrieve both the ellipsoidal parameters and the orientations of the fitted ellipsoids is applied to Multi-Angle Snowflake Camera measurements of ice particle aggregates observed in Alaska. The resulting ellipsoids have shapes closer to prolate spheroids than the oft-assumed oblate spheroids. A robust linear relationship exists between the two characteristic aspect ratios of the ellipsoids. The most probable orientation of the maximum dimension of the retrieved ellipsoids is not in the horizontal plane, and the rotational angles of the maximum dimensions in the horizontal plane are not uniform, but instead display some correlation with the wind direction at the times of the measurements. The retrieval results can be used to improve the representation of aggregates in microphysics and/or electromagnetic radiation scattering models applicable to radar and satellite measurements.
\end{abstract}

\section{Introduction}

Ice microphysical processes play important roles in both cloud and precipitation processes (e.g., Koenig and Murray 1976; Lin et al. 1983; Lord et al. 1984; Rutledge and Hobbs 1984; Dudhia 1989) and climate (e.g., Liou 1976, 1986; Hartmann and Short 1980; Ramanathan et al. 1989; Ramanathan and Collins 1991; Chen et al. 2000; Vavrus 2004; Waliser et al. 2009). As ice particles grow by vapor depositional growth and/or collisions with other particles, their sizes and fall speeds change, affecting their further growth processes (e.g., Mitchell and Heymsfield 2005; Hashino and Tripoli 2011a,b). The fall speeds of ice particles and the rates at which ice precipitates determine the lifetimes of clouds and the total amounts of precipitation produced, both of which are critical in estimating Earth energy budgets.

Modelers usually separate ice particles into different species such as pristine ice particles, aggregates, graupel,

Corresponding author: Zhiyuan Jiang, zxj113@psu.edu and hail and treat their growth processes differently (e.g., Fridlind et al. 2007; Morrison and Grabowski 2008; Thompson et al. 2008). The physics of vapor-grown ice crystals, including plates, dendrites, and columns, is comparatively well understood after decades of study (e.g., Marshall and Langleben 1954; Frank 1972; Lamb and Scott 1974; Kuroda and Lacmann 1982; Kuroda and Gonda 1984; Nelson and Knight 1998; Libbrecht 2003; Sazaki et al. 2010). However, routine measurements of ice particles reveal that most natural ice particles are not pristine but come in myriads of complex combinations with widely varying shapes (e.g., Korolev et al. 1999; Korolev and Isaac 2003; Garrett and Yuter 2014; Schmitt et al. 2016). Riming and aggregation are the two main processes transforming vapor-grown ice crystals into complex-shaped ice particles. Liquid collection by ice particles (riming) is understood less well than vapor growth of ice, but better than ice collection by ice particles (aggregation), mostly because of a lack of understanding of the collection kernel resulting from the complex flow about the colliding particles and their 
attachment processes. While the work by Westbrook et al. (2004), Connolly et al. (2012), and others have placed aggregation processes on far firmer foundations, the characteristics of aggregates produced by these processes have yet to be tested rigorously against observations. Jiang et al. (2017) showed that the threedimensionality of aggregates must be considered in the analysis of images of aggregates to eliminate biases in properties retrieved from two-dimensional images. In contrast, Jensen and Harrington (2015) were able to simultaneously match modeled mass, shape, and fall speed evolution of ice particles during riming growth against wind tunnel studies over a range of temperatures.

Of riming and aggregation, aggregation is the more common pathway to transform cloud ice to precipitation (e.g., Field and Heymsfield 2003; Mitchell and Heymsfield 2005). Yet many of the details of aggregates and the processes that govern their growth remain poorly known and, as such, cannot be represented in models. This lack of knowledge about aggregation processes may be attributed to the difficulty in observing aggregates and characterizing their shapes. Ice particle aggregates are delicate, so their shapes have to be determined while falling, implying some form of remote sensing of their properties (i.e., pictures, shadow images, radar, etc.). However, remote sensing only provides indirect information on the characteristics of aggregates and thus must be combined with other tools to enhance our understanding of aggregate properties.

Microwave remote sensing is a widely used tool to observe free-falling aggregates. To gain insights into microphysical processes, microwave measurements are often evaluated in conjunction with detailed cloudresolving models (e.g., Botta et al. 2011; Jensen et al. 2018). Such work requires transformations from model output space to microwave sensor variable space. In microphysical models, aggregates are usually represented as constant density spheres or spheroids or by several dimensional relationships involving mass, area, or aspect ratio (e.g., Fridlind et al. 2007; Morrison and Grabowski 2008; Thompson et al. 2008). But general knowledge that aggregates are not well represented by spheroids has led the microwave remote sensing community to create detailed aggregates for use in scattering calculations (e.g., Kim 2006; Liu 2008; Botta et al. 2010; Nowell et al. 2013; Tyynelä and Chandrasekar 2014; Kuo et al. 2016; Lu et al. 2016). This leads to the conundrum of having to reconcile inconsistent treatment of ice aggregates between cloud models and scattering calculations based on more realistic assumptions about them (e.g., Geer and Baordo 2014; Sieron et al. 2018). The mass, size, shape, orientation, and fall speed of aggregates are the important physical properties of aggregates that determine their growth rates and scattering properties. These physical properties have to be measured correctly and treated consistently in both microphysics and microwave scattering models. In this paper, we take a first step in documenting some of the most fundamental characteristics of aggregates: their basic shapes and orientations.

The size of an ice particle is the most frequently used physical property to link to other properties. Given a particle size, other physical properties (e.g., mass, projected area, and fall speed) are inferred using relationships reported in the literature (often obtained from independent experiments), such as mass, area, and fall speed versus size relationships (e.g., Mitchell et al. 1990; Mitchell 1996; Pruppacher and Klett 1997, p. 61). As a result, a legitimate question becomes, How well can aggregate sizes be characterized? Captured aggregates frequently disintegrate upon touching any surface, and sizes determined from two-dimensional projections of aggregates depend on the shapes and orientations of the particle (Wu and McFarquhar 2016; Jiang et al. 2017). The shape and orientation themselves must be characterized from the same projections, a problem when considering irregularly shaped objects with unknown orientations. To constrain the problem, it is common practice to treat aggregates as oblate spheroids (e.g., Matrosov et al. 2005; Kennedy and Rutledge 2011; Hogan et al. 2012), in which case, the size is known from any projection. The aspect ratio of the oblate spheroid is often taken to be the mean projected aspect ratio of 0.6 found from images (Korolev and Isaac 2003; Hogan et al. 2012; Garrett et al. 2015), particularly for radar scattering studies. However, Jiang et al. (2017) showed that the mean sampled aspect ratio of oblate spheroids will be larger than the actual aspect ratios of the spheroids, and several studies of radar scattering by aggregates have shown that assumed oblate spheroids do not fully capture the scattering properties of aggregates (e.g., Botta et al. 2011; Kneifel et al. 2011; Leinonen et al. 2012; Kulie et al. 2014; Ori et al. 2014; Tyynelä and Chandrasekar 2014).

Another important attribute of aggregates is their three-dimensional orientation as they fall. The orientation of an individual particle determines its horizontal cross-sectional area and thus fall speed (given mass), which, in turn, determines how the aggregate will interact with other particles. Microwave remote sensors sample a huge number of aggregates, each with its own orientation vector and with these orientation vectors often assumed to be a Gaussian distribution of the maximum dimension of the aggregate about the horizontal plane. Complex-shaped aggregates may gently float down or fall with rotational and/or spiral patterns when their mass centers are different from their 
geometrical centers (Kajikawa 1982, 1989; Mitra et al. 1990). Such rotational patterns suggest that the orientation vector directions vary widely in three-dimensional space, making them difficult to retrieve from single image in situ measurements, just as for particle size.

Multiple two-dimensional projections of the same ice particle alleviate some of the difficulties in retrieving particle shape and orientation. Kleinkort et al. (2017) presented a technique to perform three-dimensional shape reconstructions from five simultaneous images of a single particle. In this study, we present results from a Multi-Angle Snowflake Camera (MASC; Garrett et al. 2012), which records three simultaneous twodimensional projections of the same particle. In keeping with the desire to maintain an analytical shape, but with more degrees of freedom than a spheroid, we adopted ellipsoids to represent aggregates. The shape and orientation of an ellipsoid are specified by six parameters-three semiaxes $a, b$, and $c$ for shape and three Euler rotation angles $\alpha, \beta$, and $\gamma$ for orientation. We developed an algorithm based on gradient descent (GD) and a genetic algorithm (GA) to retrieve the six parameters of an ellipsoid from three projections of the same aggregate. (More details on the retrieval algorithm are contained in the appendix.) Retrieval results from aggregate images collected by a MASC deployed on the north slope of Alaska are shown as the main content of this paper.

\section{Data analysis}

We analyzed MASC images of aggregates taken during snow events at Barrow, Alaska (now known as Utqiagivik), during April and May 2014, and Oliktok Point, Alaska, from March 2016 to December 2017. At Barrow, the MASC was deployed on top of an instrument shelter, approximately $6 \mathrm{~m}$ above the surrounding surface, with its central camera facing into the prevailing wind (east-northeasterly). At Oliktok Point, the MASC was deployed in the center of a double Belfort Alter fence (diameters 1.2 and $2.4 \mathrm{~m}$ ), $20 \mathrm{~cm}$ below the fence top, again with the central camera facing into the prevailing east-northeasterly wind (F. Helsel 2018, personal communication).

The initial image processing followed Garrett et al. (2012), but only particles with three images, one from each of the three cameras, were retained for further analysis. As a next step, we followed Huang et al. (2010), requiring that the vertical dimension of the aggregate be approximately the same in all three images. This requirement ensured that all the cameras recorded the entirety of the aggregate. For the images that we analyzed the maximum vertical dimension across the three images had to be smaller than 1.2 times the minimum vertical dimension of the aggregate over the three images. This condition ensured that all three cameras, which are located in the same horizontal plane, captured sufficient shape and orientation information to enable retrievals with quantifiable errors. Finally, a size threshold of $5 \mathrm{~mm}$ was used to eliminate smaller pristine ice crystals from the study. Graupel are excluded by visual inspection of all particles that met the size criteria. A total of 950 aggregates out of more than 45000 particles passed these selection criteria. Note that riming was not intentionally ruled out so that rimed aggregates were included in the dataset as well.

The retrieval algorithm used GD, a GA, and a combination of the two methods (GA-GD) to minimize the differences between the two-dimensional projected ellipses from the retrieved ellipsoid and ellipses fitted to the two-dimensional projections of the ice particle. (See appendix for details.) Three semiaxes $a, b$, and $c$ (for shape) and three Euler rotation angles $\alpha, \beta$, and $\gamma$ (for orientation) of the ellipsoid were retrieved as the output. The Euler rotation angles are specified in the appendix of Jiang et al. (2017).

The largest source of uncertainty in the retrievals came from the differences between the projected ellipses of the ellipsoid fitted about the aggregate (the "truth") and the ellipses fitted to the projections of the aggregate (the input to the retrieval). These systematic differences were represented by the final value of the cost function in the fit (see appendix), which we used to indicate the accuracy of the retrieval.

\section{Results}

We ordered the semiaxes $a, b$, and $c$ of the retrieved ellipsoids so that $a>b>c$. Figure 1 shows twodimensional frequency of occurrence histograms of the two retrieved aspect ratios $b / a$ and $c / a$ for all 950 aggregates. Because of the semiaxes ordering and the definitions for the aspect ratios, only the lower-right triangle of each panel contains solutions. In these histograms, the red diagonal line represents prolate spheroids $(a>b=c)$ and the green vertical line oblate spheroids $(a=b>c)$. The first observation from these figures is that the MASC-observed aggregates were generally ellipsoidal in shape, with the ellipsoids being closer to prolate spheroids and rarely looking like oblate spheroids. Fitting a line with $y$ intercept set to 0 to the observed aspect ratios, we obtained the dashed-dotted lines in Fig. 1. We accounted for the uncertainty resulting from the systematic error in the retrieval (see appendix) by applying an arbitrary weight of [6 (cost function)] in the linear fit to the frequency of 

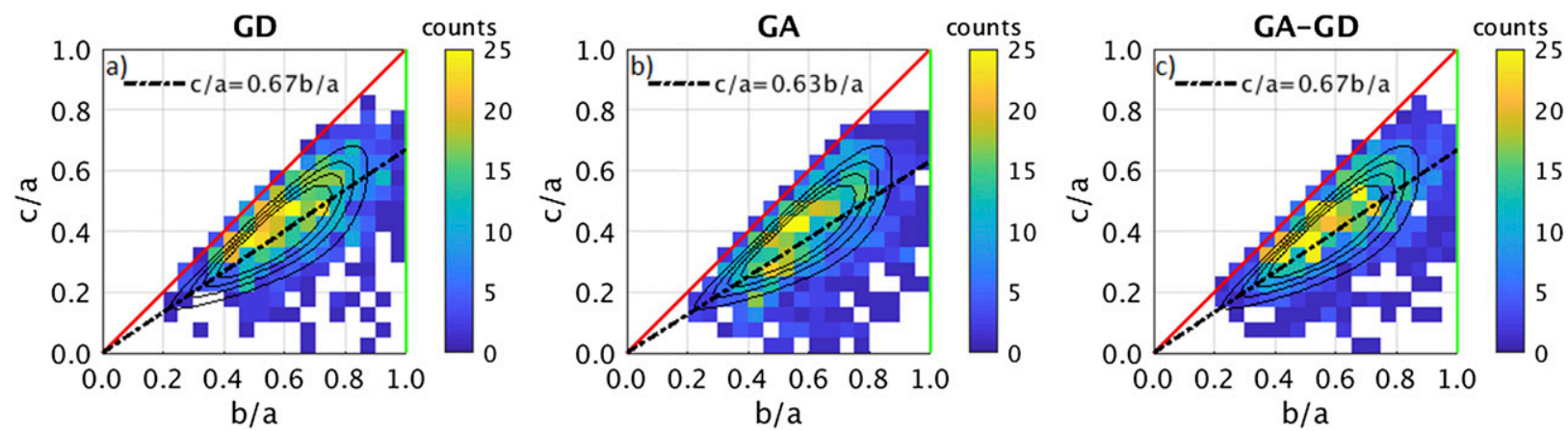

FIG. 1. Two-dimensional frequency of occurrence histograms of retrieved ellipsoidal shape (i.e., the two characteristic aspect ratios $b / a$ and $c / a$ ) of the irregular aggregates from MASC measurements obtained with (a) GD, (b) GA, and (c) GA-GD. The fitted joint PDFs of the two aspect ratios are shown as black contours.

occurrences as a function of the two retrieved aspect ratios. The three retrieval methods all gave similar fitted linear relationships between the two aspect ratios, with the smaller aspect ratio about $2 / 3$ of the larger one. This result suggests that aggregates may be parameterized better as ellipsoids with a linear relation between the two aspect ratios than as the often-used sphere or oblate spheroid.

This change in understanding of aggregate shapes has consequences on modeled properties of aggregates. One immediate impact is on aggregate fall speed calculations. The fall speed of any particle depends on the ratio of particle mass to effective cross-sectional area (Lamb and Verlinde 2011, p. 383). Although the effective crosssectional area is a function of the particle fall speed through the drag coefficient, Böhm [1989, their Eq. (10)] shows that the fall speed is inversely proportional to the square root of the particle cross-sectional area $A_{c}$. The projected area of the centroid ellipsoid shape $(b / a=0.60$ and $c / a=0.40)$ can differ by $40 \%-60 \%$ from that of an oblate spheroid with 0.6 aspect ratio, resulting in a $20 \%-40 \%$ difference in fall speed. We also examined the ice-covered area fraction $\left(A_{i} / A_{c}\right)$ for each of the fitted ellipses, where $A_{i}$ is the area within $A_{c}$ containing ice. The area fraction distributions are consistent among the three cameras, with a mean fraction of 0.82 . The consistency in area fractions suggest that it is reasonable to assume that this ratio will hold for any projection of the ellipsoid, allowing for estimates of the actual cross section of the aggregate. Together, the changes in the fall speed and the actual cross-sectional area impact the further collisional growth of the aggregate, and thus precipitation rates.

To characterize the uncertainty of the fitted relationship between the two aspect ratios, the joint probability density function of the two aspect ratios was assumed to follow a bivariate beta distribution [Nadarajah and Kotz 2005, their Eq. (4)]:

$$
\begin{aligned}
\operatorname{PDF}\left(\frac{b}{a}, \frac{c}{a} \mid p_{1}, p_{2}, p_{3}\right) \\
=\frac{\left(\frac{c}{a}\right)^{p_{1}+p_{2}-1}\left(\frac{b}{a}-\frac{c}{a}\right)^{p_{3}-1}\left(\frac{b}{a}\right)^{-p_{2}-p_{3}}\left(1-\frac{b}{a}\right)^{p_{2}-1}}{B\left(p_{1}, p_{2}\right) B\left(p_{1}+p_{2}, p_{3}\right)},
\end{aligned}
$$

where $p_{1}, p_{2}$, and $p_{3}$ are parameters to be determined from the retrievals and $B$ is the beta function. The analytical expression for the product moments of the two aspect ratios is

$$
E\left[\left(\frac{c}{a}\right)^{m}\left(\frac{b}{a}\right)^{n}\right]=\frac{B\left(m+n+p_{1}, p_{2}\right) B\left(m+p_{1}+p_{2}, p_{3}\right)}{B\left(p_{1}, p_{2}\right) B\left(p_{1}+p_{2}, p_{3}\right)} .
$$

The three parameters are estimated from the product moments $E(b / a), E(c / a)$, and $E[(b / a)(c / a)]$ of the two aspect ratios as

$$
\begin{aligned}
& p_{1}=\frac{E\left(\frac{b}{a}\right) E\left(\frac{c}{a}\right)-E\left(\frac{b}{a}\right) E\left(\frac{b}{a} \frac{c}{a}\right)}{E\left(\frac{b}{a} \frac{c}{a}\right)-E\left(\frac{b}{a}\right) E\left(\frac{c}{a}\right)}, \\
& p_{2}=p_{1}\left[\frac{1}{E\left(\frac{b}{a}\right)}-1\right], \\
& p_{3}=p_{1}\left[\frac{1}{E\left(\frac{c}{a}\right)}-1\right]-p_{2} .
\end{aligned}
$$

By doing so, the three product moments, including the centroid of the distribution, that is, $E(b / a)$ and $E(c / a)$, are conserved. The three parameters obtained from the current measurements are

$$
p_{1}=5.9691, \quad p_{2}=3.9714, \quad p_{3}=5.0479 \text {. }
$$




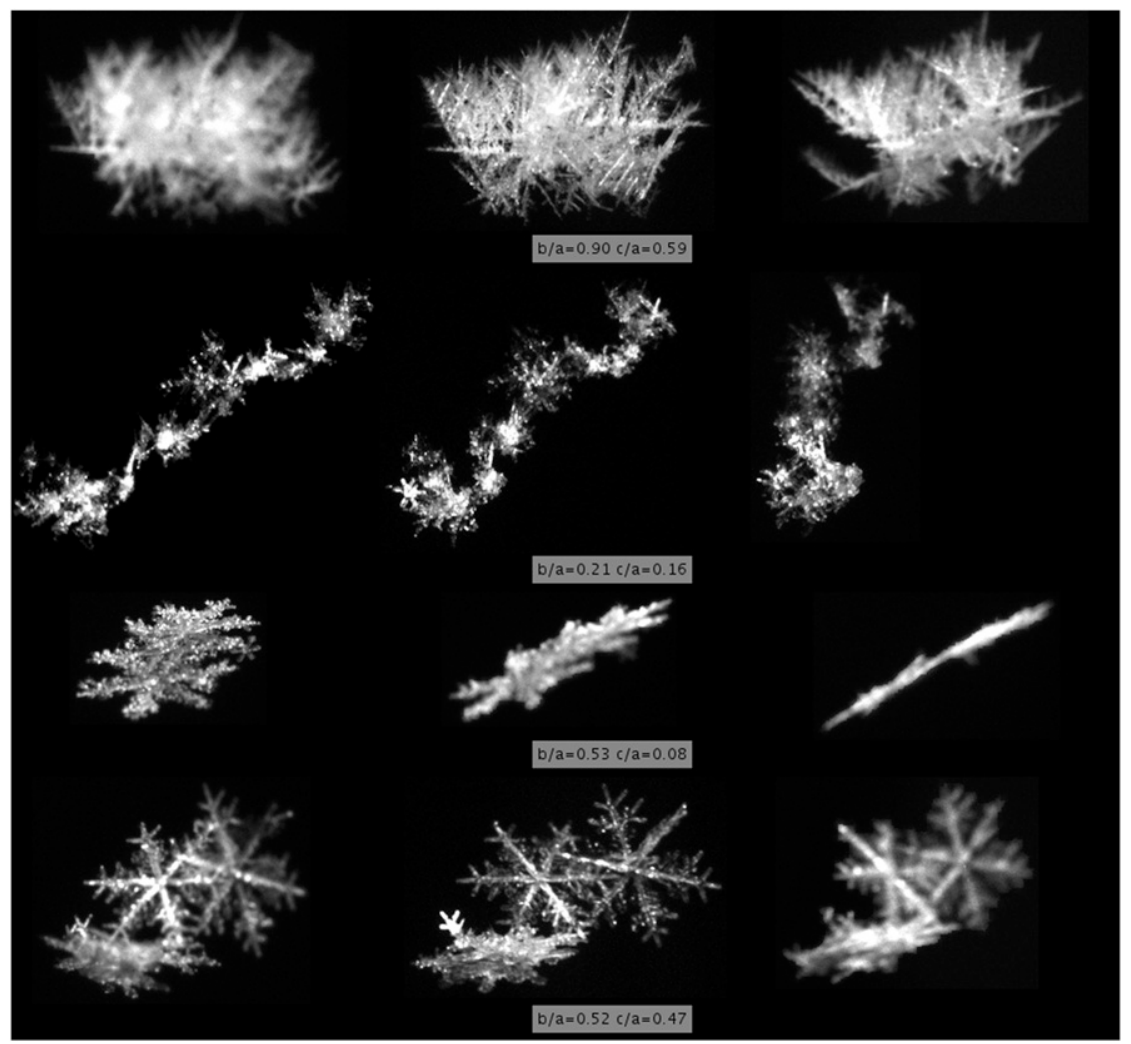

FIG. 2. Example MASC images of aggregates along with the two retrieved aspect ratios for them. The spatial scales for the images are identical, allowing their relative sizes to be easily compared. Each row represents the same aggregate with the three images in a row representing distinct viewing directions.

The advantage of using the bivariate beta distribution is that it requires $c / a \leq b / a$, consistent with our convention for characterizing ellipsoidal shape. Nadarajah and Kotz (2005) showed that the conditional probability density distribution is a beta distribution, the conditional moments of which are known analytical expressions. These analytical expressions facilitate development of parameterization schemes for aggregate processes.

Example images from MASC measurements of different ellipsoidal shapes are given in Fig. 2 together with the two retrieved aspect ratios (averaged over the three algorithms GD, GA, and GA-GD) for each of them. Because each aggregate experiences unique microphysical growth processes, different aggregates will have different numbers of monomers of different types and sizes, with different attachment orientations. The more general ellipsoidal shape assumed in our study allows us to capture the greater variety of aggregate shapes revealed in Fig. 2 better than the typically assumed spheroidal shape.

Westbrook et al. (2004) showed that their (simulated) aggregation process converges to a single set of geometric relationships, as viewed in the context of a single two-dimensional projection, independent of monomer characteristics as the number of monomers increases. Our set of images represents aggregates typical of shallow mixed-phase clouds in the Arctic. Figure 1 shows that these end products of aggregation processes assume a wide distribution of three-dimensional shapes, suggesting that the Westbrook et al. (2004) assumption may not be appropriate, at least for the cloud types represented in this study.

The orientation vector of a spheroid is two-dimensional by definition (e.g., Metcalf 1988) and is usually specified by the elevation and azimuth angles of its symmetry axis or the axis along the maximum dimension. It is often assumed that particles have a preferred orientation with their maximum dimension in the horizontal plane (e.g., Sassen 1987; Klett 1995; Ryzhkov et al. 2011). For ellipsoids, the orientation vector is three-dimensional and specified by the Euler angles (e.g., Jiang et al. 2017). However, it is not intuitive how the Euler angles are related to the orientation of a specific dimension (e.g., maximum dimension) of the ellipsoid. Here, we characterized the orientation of the ellipsoids by the elevation (EL) angle, defined as the angle between the 
horizontal and the maximum dimension. These EL angles may be considered as a proxy for the canting angle discussed in radar papers. These angles were calculated from the Euler angles as

$$
\mathrm{EL}=\cot ^{-1}\left(\frac{\sqrt{\cos ^{2} \alpha+\cos ^{2} \beta \sin ^{2} \alpha}}{|\sin \alpha \sin \beta|}\right) .
$$

The probability density functions of the retrieved elevation angles are shown in Fig. 3.

The elevation angle represents how much the maximum dimension deviates from the horizontal plane. The often-assumed maximum dimension preferentially in the horizontal plane corresponds to an elevation angle equal to $0^{\circ}$. However, the elevation angle probability density function (black dotted-dashed line in Fig. 3a) peaks at $\sim 10^{\circ}$, indicating that the sampled aggregates did not fall with their maximum dimensions preferentially in the horizontal plane. The aggregates favored a deviation from the horizontal plane over a horizontal orientation in these MASC measurements. This is physically plausible. Preferred horizontal orientation of the maximum dimension assumes that the distribution of mass within an ice particle is symmetrical; however, the stochastic nature of aggregation suggests that it is unrealistic to assume that the center of mass of an aggregate corresponds with its geometric center.

Because of the design of the MASC, higher wind speeds create stronger turbulence near the MASC (Garrett et al. 2015). We further subsampled the aggregates according to the environmental wind speed $w_{s}$ at their time of observation (within the same minute). We divided the two sets of retrieved triplets into three groups (Fig. 3a): $w_{s}<3.0 \mathrm{~m} \mathrm{~s}^{-1}$ (226 aggregates), $3.0 \leq$ $w_{s} \leq 7.0 \mathrm{~m} \mathrm{~s}^{-1}$ (342 aggregates), and $w_{s}>7.0 \mathrm{~m} \mathrm{~s}^{-1}$ (134 aggregates). For larger wind speeds $\left(>7.5 \mathrm{~m} \mathrm{~s}^{-1}\right)$, the distribution of elevation angles was relatively flat between $0^{\circ}$ and $70^{\circ}$, whereas for weak winds $\left(<3.0 \mathrm{~m} \mathrm{~s}^{-1}\right)$, the distribution was much narrower with a peak at $\sim 15^{\circ}$. The dependence of elevation angle distribution on wind speed still exists for observations without a fence (Fig. 3b), even though the sample size is small (only 71 aggregates). This dependence on wind speed suggests that the orientations of the aggregates may be influenced by turbulence generated by the instrument and/or shear across the top of the fence surrounding the MASC or the shear in the wind speed near the surface. However, it is also possible that aggregate orientation has a natural dependence on wind speed and independent of instrument impacts on it. In general, the wind speed gradient near the surface is larger with larger wind speed. With the existence of velocity gradients, even
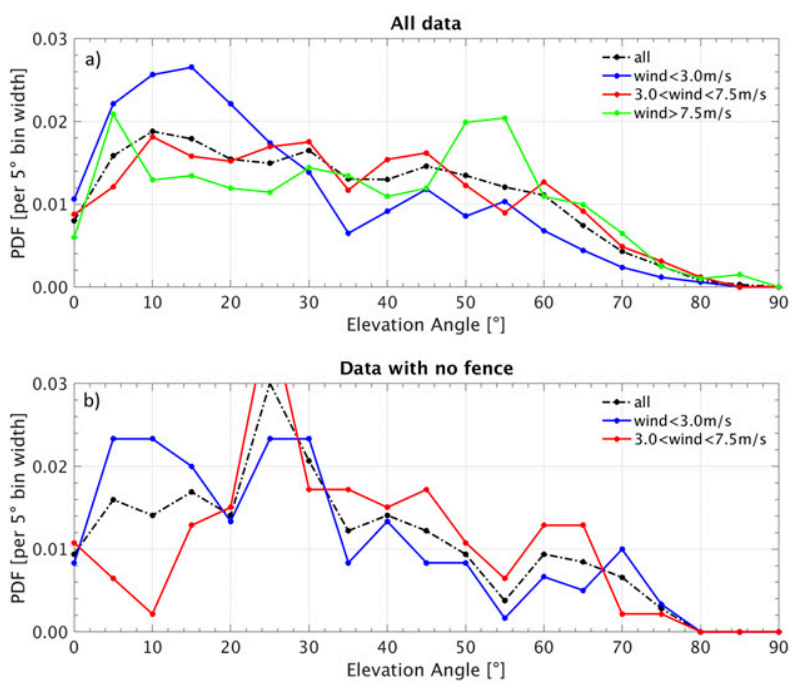

FIG. 3. PDFs of the retrieved elevation angles for (a) all of aggregates in the study and (b) the 71 aggregates in the study collected without a fence around the MASC when the measurements were made. The elevation angles for the aggregates are illustrated by the black dotted-dashed line, whereas those for aggregates observed at wind speeds less than $3.0 \mathrm{~m} \mathrm{~s}^{-1}$, between 3.0 and $7.5 \mathrm{~m} \mathrm{~s}^{-1}$, and larger than $7.5 \mathrm{~m} \mathrm{~s}^{-1}$ are illustrated by the blue, red, and green lines, respectively.

symmetric spheroidal particles with homogeneous density have preferential alignments (e.g., Bernstein and Shapiro 1994; Voth and Soldati 2017). The peak mode and spread of the orientation distribution depends on the velocity gradient. According to Berstein and Shapiro (1994), the orientation distribution is not only affected by the flow velocity gradient, but also the particle rotational diffusion, which maintains or restores the equilibrium statistical distribution of the overall orientation of particles. For nonhomogeneous ice aggregates with complex rotational diffusion, the orientation distribution can be more complex compared with homogeneous spheroidal particles.

We also investigated the distribution of the rotation angles of the maximum dimensions (projected onto the horizontal plane) of the aggregates. This distribution generally is assumed to be uniform. Our results (not shown) suggest that the aggregates preferentially aligned their maximum dimensions normal to the predominant wind direction, although comparisons to equal-in-number random samples from a uniform rotation distribution suggested that our sample size is too small to make a firm conclusion regarding this result. A torque, due to a horizontal wind-induced drag force, applied to particles with mismatched geometric and mass centers will cause the particles to rotate. However, at this point, we are unable to conclude whether or not the retrieved preference in azimuthal orientation is physical or the result of undersampling. 


\section{Summary and conclusions}

Aggregation is one of the dominant cold cloud precipitation production processes, yet few observations exist to inform the representation of this important process in cloud models. Progress on this front requires consistent treatment of aggregate properties in forward models of observation systems (e.g., radar and satellites) and cloud microphysical models. Currently, most forward models treat aggregates as oblate spheroids with an aspect ratio of 0.6. Such spheroidal models do not fully capture the scattering properties of ice aggregates (e.g., Liu 2008; Botta et al. 2010). In the microphysical parameterizations within cloud models, aggregate properties are mostly represented through dimensional relationships retrieved from observations, some of which are aimed to capture shape effects (e.g., Thompson et al. 2008). To allow more flexibility in representing irregular shapes, while maintaining the simplicity necessary to be represented in microphysical parameterizations, ellipsoids are proposed to represent aggregates in this study.

The bulk of our knowledge of atmospheric ice particle shapes has been derived from single two-dimensional images of particles. The assumption of spheroidal shapes, valid for pristine vapor-grown ice particles, was used in the analyses of these images. This practice was subsequently extended to analyses of irregular particles. The impact of orientation on the images of spheroids and/or irregular particles has been acknowledged but then, with few exceptions, largely ignored. This was perhaps the result of a single image not containing sufficient information to draw conclusions about both shape and orientation, and yet only single images have been largely available over the past couple of decades. Here, we exploit the recent availability of novel instruments that record multiple images of the same ice particle from different view directions to develop a retrieval algorithm of both particle shape and orientation.

Because spheroids are included as special cases in the general category of ellipsoids, our retrieval results allow us to examine the often-used assumption of representing aggregates with spheroidal shapes. We show that our sample of 950 aggregates are better represented by ellipsoids than spheroids, with a robust linear relationship between the two aspect ratios of ellipsoids. We characterized the spread in the two aspect ratios using a bivariate beta distribution. This retrieved distribution of aspect ratios is used to infer values and uncertainties in other parameters, for example, horizontally projected cross-sectional areas needed for fall-speed calculations. The analytical formula of the distribution, including the product moments and conditional probability distribution, is amenable to use in parameterization schemes.
The work presented here is a step in the same direction as the earlier work of Kleinkort et al. (2017) and is applicable to all MASC measurements as well as other multiview instruments such as the 3-View Cloud Particle Imager. Simultaneous mass measurements of individual aggregates must be made in conjunction with shape and orientation measurements to complete the analytical description of aggregates necessary for microphysical model development; unfortunately, simultaneous measurements of shape, orientation, and mass for individual aggregates do not currently exist. In lieu of such direct measurements, retrievals of effective density based on MASC measurements of fall speed and retrievals of shape and orientation become an avenue for making additional progress on this problem (e.g., Huang et al. 2015).

Most of the aggregates in the sample of 950 for this study were not aligned with their maximum dimension in the horizontal plane. The distribution of the angles between the horizontal plane and the maximum dimension derived in this study is quite distinct from those derived from single images (e.g., Gergely et al. 2017). The sensitivity of the canting angle distribution to the wind speed at time of measurement suggests that careful attention must be given to the deployment of the MASC. Without a fence, the sampling efficiency of the instrument is low and a strong function of the prevailing wind speed. Thériault et al. (2012) showed that using a fence may introduce shape and size biases in the sampled population, and our study suggests fences may also introduce wind speed-dependent orientation biases.

Heymsfield et al. (2004) showed that the projected areas of ice particles obtained from images are related to the density of the ice particles. It warrants further study to determine how (or if) a measure of projected area for these irregularly shaped aggregates may be related to mass density. There is an urgent need in the community to add individual particle mass measurements together with multiple view imaging instruments.

Acknowledgments. The authors thank Dr. T. J. Garrett from the Department of Atmospheric Sciences at the University of Utah for sharing the MASC preprocessing code and Dr. G. S. Young and the Ph.D. student E. L. Dunnavan from the Department of Meteorology and Atmospheric Science at The Pennsylvania State University for discussions regarding the retrieval algorithm and the bivariate beta distribution that we used in the study. This research was supported by the U.S. Department of Energy's Atmospheric System Research, an Office of Science, Office of Biological and Environmental Research program, under Grant DE-SC0013953. The MASC image data are available from the DOE ARM 
archive (www.archive.arm.gov), and the 950 triplet images and retrieval results can be downloaded online (https://doi.org/10.18113/S1BH15).

\section{APPENDIX}

\section{Shape and Orientation Retrieval Algorithm}

We describe in detail the methodology to retrieve the shape and orientation of an ellipsoid from three twodimensional projections similar to those from the MultiAngle Snowflake Camera (MASC) and show results obtained by applying the retrieval to artificially generated irregular aggregates.

An ellipsoid with arbitrary orientation is specified by six variables: the three semiaxes $a, b$, and $c$ and the three Euler rotation angles $\alpha, \beta$, and $\gamma$. A single twodimensional projection yields three independent observables: the two semiaxes $R_{a}$ and $R_{b}$ of the projected ellipse and the angle $\theta$ of its maximum dimension relative to the horizontal axis [positive (negative) when the maximum dimension is in first and third (second and fourth) quadrants; Fig. A1]. Note that the angle $\theta$ is different from Garrett et al. (2015) as they use the absolute value of $\theta$ as we defined it here. The MASC's three viewing directions yield a total of nine observables from which to retrieve the six defining shape and orientation variables of the ellipsoid. Jiang et al. (2017) derived analytical expressions for the observables in terms of the six parameters describing an ellipsoid and the three viewing directions. These nine expressions, one for each of the observables, constitute the forward model in our retrieval. We employ both gradient descent and genetic algorithms to determine the six ellipsoidal parameters from the nine observables.

Gradient descent (GD) is an iterative algorithm used to find the minimum of a function by moving the solution down the gradient from its current location in the solution space. Let $\mathbf{f}$ represent the forward model from the ellipsoidal parameter space

$$
\mathbf{x}=(a, b, c, \alpha, \beta, \gamma)^{\mathrm{T}}
$$

to observation space

$$
\mathbf{y}=\left(R_{a}^{1}, R_{a}^{2}, R_{a}^{3}, R_{b}^{1}, R_{b}^{2}, R_{b}^{3}, \theta^{1}, \theta^{2}, \theta^{3}\right)^{\mathrm{T}},
$$

where the superscripts indicate the three different projections. Ideally, we want to find $\mathbf{x}$ such that $\mathbf{f}(\mathbf{x})=\mathbf{y}$. However, being unable to solve for $\mathbf{x}=\mathbf{f}^{-1}(\mathbf{y})$ analytically, we instead search for a solution $\mathbf{x}^{*}$ that minimizes the cost function

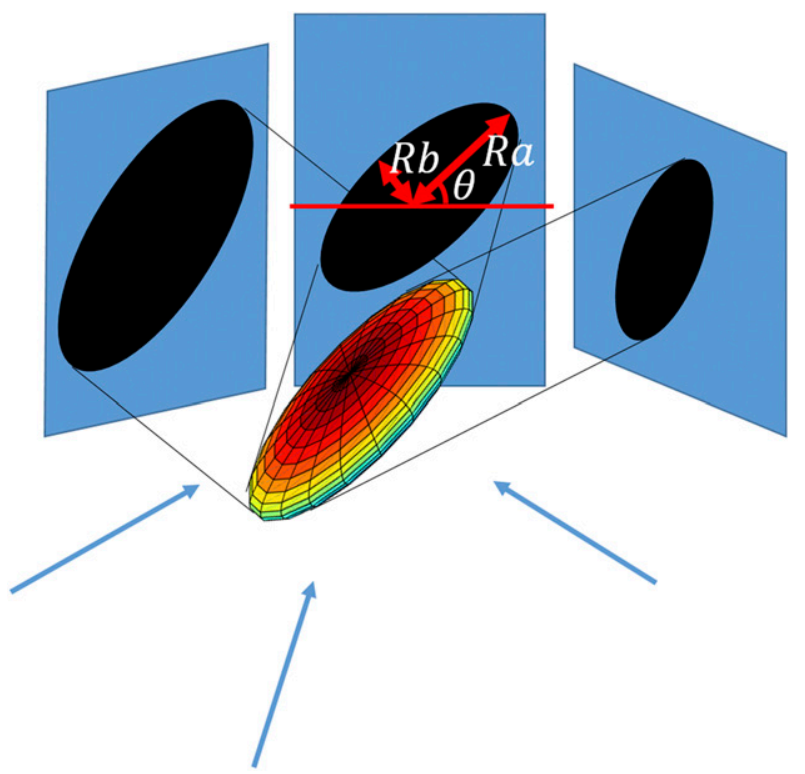

FIG. A1. A schematic figure showing the observables that are obtained from two-dimensional projections of an ellipsoid. This schematic is based on the three MASC viewing directions and the nine observables that are provided by them.

$$
\left|\mathbf{f}\left(\mathbf{x}^{*}\right)-\mathbf{y}\right|=\sqrt{\left[\mathbf{f}\left(\mathbf{x}^{*}\right)-\mathbf{y}\right]^{\mathrm{T}} \times\left[\mathbf{f}\left(\mathbf{x}^{*}\right)-\mathbf{y}\right]} .
$$

Starting from an initial guess $\mathbf{x}_{0}$, the estimate of $\mathbf{x}^{*}$ is updated at every step using

$$
\mathbf{x}_{n+1}=\mathbf{x}_{n}-r \mathbf{J}_{\mathbf{f}}\left(\mathbf{x}_{n}\right)\left[\mathbf{f}\left(\mathbf{x}_{n}\right)-\mathbf{y}\right]+0.1\left(\mathbf{x}_{n}-\mathbf{x}_{n-1}\right),
$$

where $r$ is the learning rate ( 0.15 in this study) and $\mathbf{J}_{\mathbf{f}}$ is the Jacobian matrix of the forward model. One-tenth of the previous step is added to overcome local minima and speed up convergence. Stopping criteria are the tolerance of error (0.001), that is, the value of the cost function below which the search stopped, and the total number of iterations (1000). We use several initial guesses spread out in the solution space to avoid local minima. The final solution is selected by the $\mathbf{y}$ that leads to the lowest achieved cost function over all starting conditions.

The space covered by the initial guesses $\mathbf{x}_{0}$ is constrained by the observations $\mathbf{y}$. The semimajor axis $a$ of the ellipsoid is at least as large as the maximum semimajor axis of the three projected ellipses, or $\max \left(R_{b}^{1}, R_{b}^{2}, R_{b}^{3}\right)$, which is used as the initial guess of $a$. The semiaxis $b$ of the ellipsoid ranges between the maximum semiminor axis $b$ of the three projected ellipses, or $\max \left(R_{b}^{1}, R_{b}^{2}, R_{b}^{3}\right)$, and the minimum semimajor axis $a$ of the three projected ellipses, or $\min \left(R_{a}^{1}, R_{a}^{2}, R_{a}^{3}\right)$. We select four evenly distributed initial guess values for $b$ across this range. The semiaxis $c$ of the ellipsoid is at most as large as the minimum semiminor axis $b$ of the three 
projected ellipses, or $\min \left(R_{b}^{1}, R_{b}^{2}, R_{b}^{3}\right)$, which is used as the initial guess of $c$.

There are not as many constraints for orientation as for size. Prolate and oblate spheroidal shapes allow for accurate orientation retrieval (Jiang 2016). Because prolate spheroidal orientation retrieval is more accurate than oblate spheroidal orientation retrieval when obtained within a general ellipsoidal shape retrieval framework (Fig. 5.8 in Jiang 2016), prolate spheroidal orientation retrieval is used to obtain the initial guesses of $\alpha$ and $\gamma$. Initial guesses of $\beta$ start at the three values of $45^{\circ}, 90^{\circ}$, and $135^{\circ}$. Note that radians are used for the units of the angles in the calculation of the cost function to keep the contributions of the angle and shape parameters to the cost function approximately equal. Therefore, 12 initial guesses of $\mathbf{x}_{0}$ ( 4 different initial guesses for $b$ times 3 different initial guesses of $\beta$ ) are used to avoid local minima in the cost function.

The projection orientation angle $\theta$ in the forward model f is not continuous because it is constrained to fall between $-90^{\circ}$ and $90^{\circ}$. A constraint is applied limiting the change in $\theta$ to less than $90^{\circ}$ in the calculation of the relevant derivative in the gradient descent. If the change is larger than $90^{\circ}$, it is replaced by $180^{\circ}$ minus the change. Even though this procedure does not eliminate the discontinuity in the cost function, the constraint does make the derivative of the orientation angle small, thus avoiding big changes when updating $\mathbf{x}_{n}$.

Genetic algorithms (GA) are also iterative. The GA that we used minimizes the cost function by simulating a natural selection process. The first generation of the population is taken to be the same as the initial guesses used in the GD method. Members of the population with smaller cost functions have higher probabilities to pass part of their characteristics to the next generation and a higher chance of being selected as parents. We use the tournament selection method to select parents (Goldberg and Deb 1991).

In our implementation of this method, the best individual from four randomly selected members of the population is taken as a parent. The next generation is produced from two parents whose offspring results from a linear combination of the parents obtained by randomly selecting a point on the segment connecting the parents. This process is called crossover. The segment can be shrunk or extended by a factor called the crossover scale ( 2 in this study). After crossover, each element of the child is allowed to mutate with a prescribed mutation probability. The mutation probability for each element of $\mathbf{x}_{n}$ can be tuned separately depending on how often we want that element to mutate. We used mutation probabilities of 0.1 for shape parameters and 1 for orientation parameters. Each new generation is the final result of the selection, crossover, and mutation processes. Stopping criteria are a maximum number of 600 generations and an average relative change of $10^{-16}$ in the cost function over 50 generations.
It is not obvious which algorithm is more effective in our application. Therefore, both methods are used in this study. Haupt and Haupt (1998) suggested that these two methods can be combined by first using the genetic algorithm to find several starting points for the gradient descent method such that local minima can be avoided and then using the gradient descent to further improve retrieval accuracy. Thus a third method, a combined genetic algorithm-gradient descent method (GA-GD), is also used. Tests of the three methods with randomly generated ellipsoids show that the mean retrieval bias is less than $5 \%$ in semiaxes and less than $10^{\circ}$ in Euler angles.

Artificial irregular aggregates from Schmitt and Heymsfield (2010) are used to further evaluate the retrieval algorithm. Details on how the aggregates are generated can be found in that paper, but are quickly summarized here. An aggregate starts with a randomly oriented ice crystal. Additional randomly oriented ice crystals are added to the aggregate at a random location one at a time. The aggregates are composed of 20 hexagonal crystals, each with an aspect ratio of either 0.1 (thin plate), 0.4 (thick plate), 1.25 (isometric column), or 10 (column). All crystals in each aggregate have the same aspect ratio. In this study, the size of each crystal is allowed to change by a random factor between 0.5 and 2, which is different from Schmitt and Heymsfield (2010). Examples of the artificial aggregates are shown in Fig. A2. A total of 44 aggregates were produced to test the retrieval algorithm. For each aggregate, four sets of three images using the MASC viewing geometry were generated with orientations drawn from uniform distributions for each of the Euler angles. Note that the size of each aggregate is scaled to be about $5 \mathrm{~mm}$.

Because aggregates have complex shapes, and therefore, complex-shaped projections, application of the retrieval algorithm requires fitting ellipses to the projections of the aggregate. The fitted ellipse (we use the MATLAB function "regionprops") has the same normalized second central moments as the image (e.g., $\mathrm{Hu}$ 1962). The three fitted ellipses are used to retrieve the ellipsoidal shape and orientation that best represents the aggregate. We evaluate retrieval accuracy by comparing the ellipsoid retrieved from the three fitted ellipses with the ellipsoid fitted directly to the artificial (threedimensional) aggregate itself. This fitting introduces another degree of uncertainty in the retrieval because the ellipses fitted to the two-dimensional projections of the aggregate will not necessarily correspond to the best fit ellipsoid to the (three-dimensional) aggregate itself. Even though we treat the best fit ellipsoid to the (threedimensional) aggregate as the "truth," the fitted ellipsoid may be different based on different fitting algorithms. 


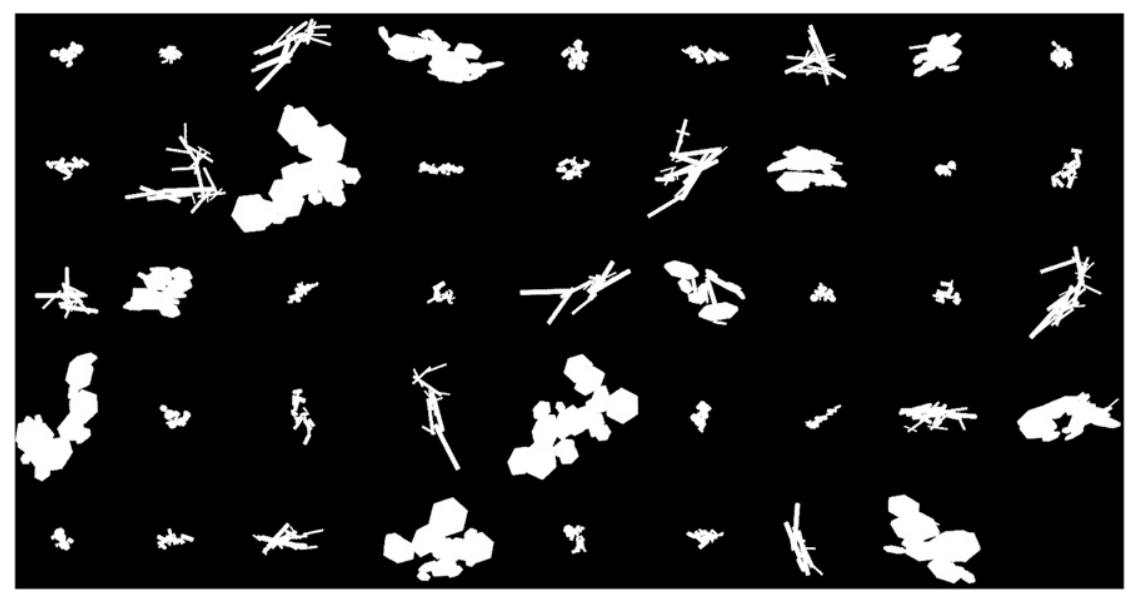

FIG. A2. Examples of artificial irregular aggregates used to evaluate the retrieval algorithm.

The procedure for fitting ellipsoids to the aggregates themselves is as follows. Assume an aggregate consists of $m$ discrete elemental points with $x_{i}, y_{i}$, and $z_{i}$ representing the Cartesian coordinates of these points in the aggregate. The center of the aggregate is

$$
(\bar{x}, \bar{y}, \bar{z})=\frac{1}{m}\left(\sum_{i=1}^{m} x_{i}, \sum_{i=1}^{m} y_{i}, \sum_{i=1}^{m} z_{i}\right)
$$

A plane

$$
z-\bar{z}=A(x-\bar{x})+B(y-\bar{y})
$$

is fitted to these points that minimizes the sum of the square of the distances of these points from the plane in the $z$ direction, that is, $\sum_{i=1}^{m}\left[A\left(x_{i}-\bar{x}\right)+B\left(y_{i}-\bar{y}\right)-\right.$ $\left.\left(z_{i}-\bar{z}\right)\right]^{2}$, using linear regression. Here, $A$ and $B$ are calculated from
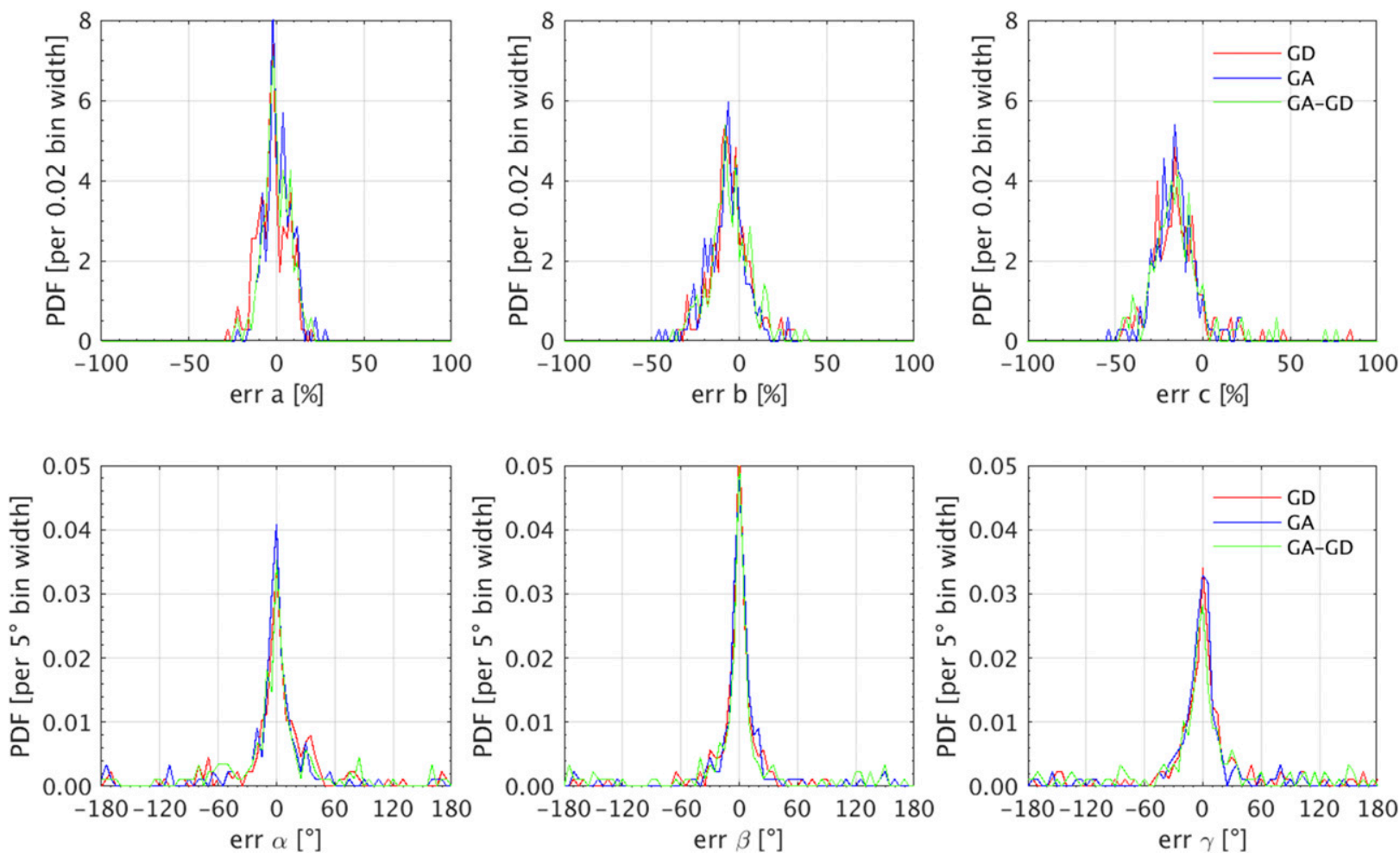

FIG. A3. PDFs of the retrieval errors for (top) the shape (i.e., $a, b$, and $c$ ) and (bottom) orientation $(\alpha, \beta$, and $\gamma$ ) parameters obtained from the GD, GA, and GA-GD methods. 

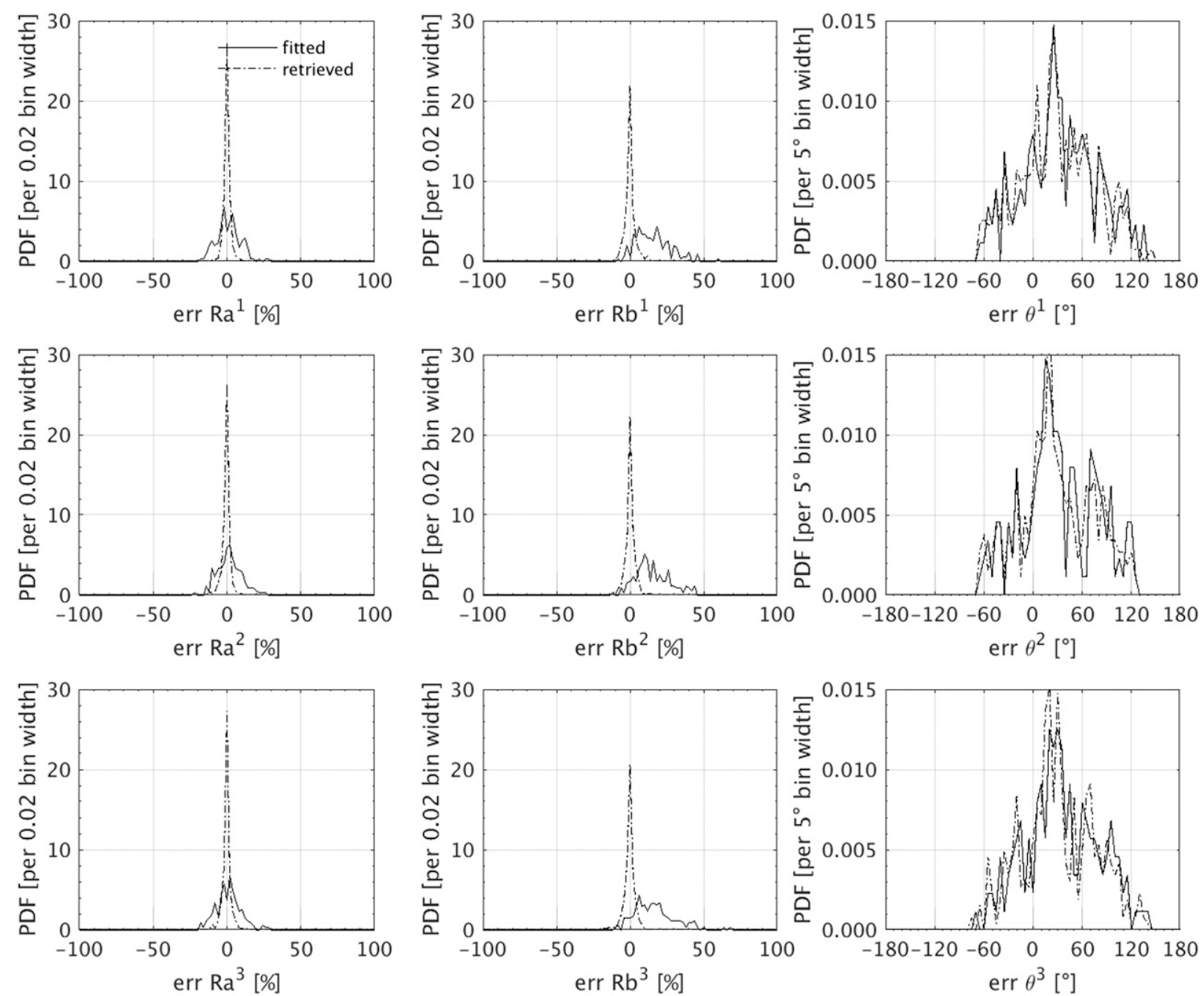

FIG. A4. PDFs of the differences between the two-dimensional projected ellipses obtained from direct projections of the aggregates themselves and those obtained from the ellipsoid fits to the aggregates.

$$
\begin{aligned}
{\left[\begin{array}{l}
A \\
B
\end{array}\right]=} & {\left[\begin{array}{cc}
\sum_{i=1}^{m}\left(x_{i}-\bar{x}\right)^{2} & \sum_{i=1}^{m}\left(x_{i}-\bar{x}\right)\left(y_{i}-\bar{y}\right) \\
\sum_{i=1}^{m}\left(x_{i}-\bar{x}\right)\left(y_{i}-\bar{y}\right) & \sum_{i=1}^{m}\left(y_{i}-\bar{y}\right)^{2}
\end{array}\right]^{-1} } \\
& \times\left[\begin{array}{l}
\sum_{i=1}^{m}\left(x_{i}-\bar{x}\right)\left(z_{i}-\bar{z}\right) \\
\sum_{i=1}^{m}\left(y_{i}-\bar{y}\right)\left(z_{i}-\bar{z}\right)
\end{array}\right]
\end{aligned}
$$

The semiaxis $b$ is assumed to be in this plane. Next, all the points in the aggregate are projected onto the fitted plane, which gives a projection of the aggregate. The ellipse fitting algorithm from the MATLAB function regionprops, the same as used to fit ellipses to the MASC images, is applied to the projection to determine the direction of the semiaxis $b$ of the ellipsoid. The particle is then projected onto a plane perpendicular to the semiaxis $b$ in order to determine the directions of semiaxes $a$ and $c$. The semiaxes $a, b$, and $c$ are half of the largest distance of the particle along each of their directions, respectively.

First, we explore retrieval errors assuming that the fitted ellipsoid represents the truth. The probability density functions (PDFs) of retrieval errors (i.e., retrieved variable value minus the truth value from the artificial aggregate) for the six ellipsoidal parameters are given in Fig. A3. The PDFs for most parameters peak near $0 \%$. However, the semiaxis $a$ is better retrieved than $b$, which has a larger spread, and $c$, which centers around $-20 \%$. The PDF of errors for the Euler angle $\beta$ has a higher peak and therefore smaller variance than 
the other two Euler angles $\alpha$ and $\gamma$. To generate the results presented in Fig. A3 we treated the ellipsoids fitted to the artificial aggregates as the truth. However, the two-dimensional projected ellipses of the (threedimensional) fitted ellipsoid do not necessarily correspond to the ellipses obtained by fits to the two-dimensional projections of the (three-dimensional) artificial aggregates. This difference leads to a systematic error that cannot be avoided.

Second, we explore the cause of the systematic error by looking at the deviations of the ellipses fitted to the projections of the aggregates from those of the projections of the fitted ellipsoids. We explore this systematic error by inspecting PDFs of the differences between the observables based on the projected ellipses of the ellipsoids fitted to the artificial aggregates and the observables based on the ellipses fitted to the projections of the artificial aggregates (Fig. A4, black lines). The differences in $R_{a}$ have a peak probability around $0 \%$, while the differences in $R_{b}$ peak near $20 \%$. The large difference in $R_{b}$ explains the retrieval bias in semiaxis $c$. For these artificial aggregates, the ellipsoid fitting algorithm tends to overestimate the $c$ axis by taking the largest distance in the $c$-axis direction.

The ellipses fitted to the projections of the artificial aggregates are subsequently input into the retrieval, which manages to retrieve ellipsoids whose projected ellipses closely (i.e., with smallest cost function) match the input (Fig. A4, black dashed lines). However, the differences between the observables $R_{a}$ and $R_{b}$ from the projected ellipses of the ellipsoids fitted to the artificial aggregates and those from the ellipses fitted to the projections of the artificial aggregates are much larger (Fig. A4, black solid lines). It is these differences that are contributing most to the retrieval errors shown in Fig. A3, where the $a$-axis length is unbiased, the $c$-axis length is biased by about $-20 \%$, and the $b$-axis length is biased by some value between $0 \%$ and $-20 \%$. This implies that the retrieval algorithm retains the information from the images, the only information provided in real observations. In contrast, ellipsoids fitted to the threedimensional artificial aggregate are less likely to have projected ellipses close to the ellipses fitted to the projections of the artificial aggregates. One interesting aspect of the projected orientation angles $\theta$ is that the two PDFs for them in the third column of panels in Fig. A4 are similar. The bulk of the systematic error results from the uncertainty introduced by the sensitivity of $\theta$ to the viewing direction for an irregularly shaped particle. All of these systematic errors suggest that the final value of the cost function may be used to identify aggregate shapes that deviate greatly from our assumed ellipsoidal shape for them.

\section{REFERENCES}

Bernstein, O., and M. Shapiro, 1994: Direct determination of the orientation distribution function of cylindrical particles immersed in laminar and turbulent shear flows. J. Aerosol Sci., 25, 113-136, https://doi.org/10.1016/0021-8502(94)90185-6.

Böhm, H. P., 1989: A general equation for the terminal fall speed of solid hydrometeors. J. Atmos. Sci., 46, 2419-2427, https://doi.org/ 10.1175/1520-0469(1989)046<2419:AGEFTT>2.0.CO;2.

Botta, G., K. Aydin, and J. Verlinde, 2010: Modeling of microwave scattering from cloud ice crystal aggregates and melting aggregates: A new approach. IEEE Geosci. Remote Sens. Lett., 7, 572-576, https://doi.org/10.1109/LGRS.2010.2041633.

,,,--- A. E. Avramov, A. S. Ackerman, A. M. Fridlind, G. M. McFarquhar, and M. Wolde, 2011: Millimeter wave scattering from ice crystals and their aggregates: Comparing cloud model simulations with $\mathrm{X}$ - and Ka-band radar measurements. J. Geophys. Res., 116, D00T04, https://doi.org/10.1029/ 2011JD015909.

Chen, T., W. B. Rossow, and Y. Zhang, 2000: Radiative effects of cloud-type variations. J. Climate, 13, 264-286, https://doi.org/ 10.1175/1520-0442(2000)013<0264:REOCTV>2.0.CO;2.

Connolly, P. J., C. Emersic, and P. R. Field, 2012: A laboratory investigation into the aggregation efficiency of small ice crystals. Atmos. Chem. Phys., 12, 2055-2076, https://doi.org/10.5194/ acp-12-2055-2012.

Dudhia, J., 1989: Numerical study of convection observed during the Winter Monsoon Experiment using a mesoscale twodimensional model. J. Atmos. Sci., 46, 3077-3107, https:// doi.org/10.1175/1520-0469(1989)046<3077:NSOCOD> 2.0.CO;2.

Field, P. R., and A. J. Heymsfield, 2003: Aggregation and scaling of ice crystal size distributions. J. Atmos. Sci., 60, 544-560, https:// doi.org/10.1175/1520-0469(2003)060<0544:AASOIC >2.0.CO;2.

Frank, F. C., 1972: An outline of nucleation theory. J. Cryst. Growth, 13-14, 154-156, https://doi.org/10.1016/0022-0248(72)90146-7.

Fridlind, A. M., A. S. Ackerman, G. McFarquhar, G. Zhang, M. R. Poellot, P. J. DeMott, A. J. Prenni, and A. J. Heymsfield, 2007: Ice properties of single-layer stratocumulus during the MixedPhase Arctic Cloud Experiment: 2. Model results. J. Geophys. Res., 112, D24202, https://doi.org/10.1029/2007JD008646.

Garrett, T. J., and S. E. Yuter, 2014: Observed influence of riming, temperature, and turbulence on the fallspeed of solid precipitation. Geophys. Res. Lett., 41, 6515-6522, https://doi.org/ 10.1002/2014GL061016.

— C. Fallgatter, K. Shkurko, and D. Howlett, 2012: Fall speed measurement and high-resolution multi-angle photography of hydrometeors in free fall. Atmos. Meas. Tech., 5, 2625-2633, https://doi.org/10.5194/amt-5-2625-2012.

—, S. E. Yuter, C. Fallgatter, K. Shkurko, S. R. Rhodes, and J. L. Endries, 2015: Orientations and aspect ratios of falling snow. Geophys. Res. Lett., 42, 4617-4622, https://doi.org/10.1002/ 2015 GL064040.

Geer, A. J., and F. Baordo, 2014: Improved scattering radiative transfer for frozen hydrometeors at microwave frequencies. Atmos. Meas. Tech., 7, 1839-1860, https://doi.org/10.5194/amt-7-1839-2014.

Gergely, M., S. J. Cooper, and T. J. Garrett, 2017: Using snowflake surface-area-to-volume ratio to model and interpret snowfall triple-frequency radar signatures. Atmos. Chem. Phys., 17, 12 011-12 030, https://doi.org/10.5194/acp-17-12011-2017.

Goldberg, D. E., and K. Deb, 1991: A comparative analysis of selection schemes used in genetic algorithms. Foundations of Genetic Algorithms, Vol. 1, Elsevier, 69-93. 
Hartmann, D. J., and D. A. Short, 1980: On the use of Earth radiation budget for study of cloud and climate. J. Atmos. Sci., 37, 1233-1250, https://doi.org/10.1175/1520-0469(1980)037<1233: OTUOER $>2.0 . \mathrm{CO} ; 2$

Hashino, T., and G. J. Tripoli, 2011a: The Spectral Ice Habit Prediction System (SHIPS). Part III: Description of the ice particle model and the habit-dependent aggregation model. J. Atmos. Sci., 68 , 1125-1141, https://doi.org/10.1175/2011JAS3666.1.

— , and — 2011b: The Spectral Ice Habit Prediction System (SHIPS). Part IV: Box model simulations of the habit-dependent aggregation process. J. Atmos. Sci., 68, 1142-1161, https://doi.org/ 10.1175/2011JAS3667.1.

Haupt, R. L., and S. E. Haupt, 1998: Practical Genetic Algorithms. Vol. 2. Wiley, 253 pp.

Heymsfield, A. J., A. Bansemer, C. Schmitt, C. Twohy, and M. R. Poellot, 2004: Effective ice particle densities derived from aircraft data. J. Atmos. Sci., 61, 982-1003, https://doi.org/ 10.1175/1520-0469(2004)061<0982:EIPDDF>2.0.CO;2.

Hogan, R. J., L. Tian, P. R. Brown, C. D. Westbrook, A. J. Heymsfield, and J. D. Eastment, 2012: Radar scattering from ice aggregates using the horizontally aligned oblate spheroid approximation. J. Appl. Meteor. Climatol., 51, 655-671, https:// doi.org/10.1175/JAMC-D-11-074.1.

Hu, M. K., 1962: Visual pattern recognition by moment invariants. IRE Trans. Inf. Theory, 8, 179-187, https://doi.org/10.1109/ TIT.1962.1057692.

Huang, G. J., V. N. Bringi, R. Cifelli, D. Hudak, and W. A. Petersen, 2010: A methodology to derive radar reflectivityliquid equivalent snow rate relations using C-band radar and a 2D video disdrometer. J. Atmos. Oceanic Technol., 27, 637651, https://doi.org/10.1175/2009JTECHA1284.1.

,,-- W. A. Petersen, L. Bliven, and D. Hudak, 2015: Use of 2D-video disdrometer to derive mean density-size and $\mathrm{Z}_{\mathrm{e}}-\mathrm{SR}$ relations: Four snow cases from the Light Precipitation Validation Experiment. Atmos. Res., 153, 34-48, https://doi.org/ 10.1016/j.atmosres.2014.07.013.

Jensen, A. A., and J. Y. Harrington, 2015: Modeling ice crystal aspect ratio evolution during riming: A single-particle growth model. J. Atmos. Sci., 72, 2569-2590, https://doi.org/10.1175/ JAS-D-14-0297.1.

,-- , and H. Morrison, 2018: Microphysical characteristics of squall-line stratiform precipitation and transition zones simulated using an ice particle property-evolving model. Mon. Wea. Rev., 146, 723-743, https://doi.org/10.1175/MWR-D-17-0215.1.

Jiang, Z., 2016: Retrieving maximum dimension, aspect ratio, and orientation of ice particles from their two-dimensional projections. M.S. thesis, Dept. of Meteorology and Atmospheric Science, The Pennsylvania State University, $61 \mathrm{pp}$.

—, M. Oue, J. Verlinde, E. E. Clothiaux, K. Aydin, G. Botta, and Y. Lu, 2017: What can we conclude about the real aspect ratios of ice particle aggregates from two-dimensional images? J. Appl. Meteor. Climatol., 56, 725-734, https://doi.org/10.1175/ JAMC-D-16-0248.1.

Kajikawa, M., 1982: Observation of the falling motion of early snow flakes: Part I. Relationship between the free-fall pattern and the number and shape of component snow crystals. J. Meteor. Soc. Japan, 60, 797-803, https://doi.org/10.2151/jmsj1965.60.2_797.

_ 1989: Observation of the falling motion of early snowflakes. Part II: On the variation of falling velocity. J. Meteor. Soc. Japan, 67, 731-738, https://doi.org/10.2151/jmsj1965.67.5_731.

Kennedy, P. C., and S. A. Rutledge, 2011: S-band dual-polarization radar observations of winter storms. J. Appl. Meteor. Climatol., 50, 844-858, https://doi.org/10.1175/2010JAMC2558.1.
Kim, M. J., 2006: Single scattering parameters of randomly oriented snow particles at microwave frequencies. J. Geophys. Res., 111, D14201, https://doi.org/10.1029/2005JD006892.

Kleinkort, C., G. Huang, V. N. Bringi, and B. M. Notaroš, 2017: Visual hull method for realistic 3D particle shape reconstruction based on high-resolution photographs of snowflakes in free fall from multiple views. J. Atmos. Oceanic Technol., 34, 679-702, https://doi.org/10.1175/JTECH-D-16-0099.1.

Klett, J. D., 1995: Orientation model for particles in turbulence. J. Atmos. Sci., 52, 2276-2285, https://doi.org/10.1175/15200469(1995)052<2276:OMFPIT>2.0.CO;2.

Kneifel, S., M. S. Kulie, and R. Bennartz, 2011: A triple-frequency approach to retrieve microphysical snowfall parameters. J. Geophys. Res., 116, D11203, https://doi.org/10.1029/2010JD015430.

Koenig, L. R., and F. W. Murray, 1976: Ice-bearing cumulus cloud evolution: Numerical simulation and general comparison against observations. J. Appl. Meteor., 15, 747-762, https://doi.org/ 10.1175/1520-0450(1976)015<0747:IBCCEN>2.0.CO;2.

Korolev, A., and G. Isaac, 2003: Roundness and aspect ratio of particles in ice clouds. J. Atmos. Sci., 60, 1795-1808, https://doi.org/ 10.1175/1520-0469(2003)060<1795:RAAROP $>2.0 . C O ; 2$.

,-- , and J. Hallett, 1999: Ice particle habits in Arctic clouds. Geophys. Res. Lett., 26, 1299-1302, https://doi.org/10.1029/ 1999GL900232.

Kulie, M. S., M. J. Hiley, R. Bennartz, S. Kneifel, and S. Tanelli, 2014: Triple-frequency radar reflectivity signatures of snow: Observations and comparisons with theoretical ice particle scattering models. J. Appl. Meteor. Climatol., 53, 1080-1098, https://doi.org/10.1175/JAMC-D-13-066.1.

Kuo, K. S., and Coauthors, 2016: The microwave radiative properties of falling snow derived from nonspherical ice particle models. Part I: An extensive database of simulated pristine crystals and aggregate particles, and their scattering properties. J. Appl. Meteor. Climatol., 55, 691-708, https://doi.org/10.1175/JAMC-D-15-0130.1.

Kuroda, T., and R. Lacmann, 1982: Growth kinetics of ice from the vapour phase and its growth forms. J. Cryst. Growth, 56, 189205, https://doi.org/10.1016/0022-0248(82)90028-8.

_ and T. Gonda, 1984: Rate determining processes of growth of ice crystals from the vapour phase. Part II: Investigation of surface kinetic process. J. Meteor. Soc. Japan, 62, 563-572, https://doi.org/10.2151/jmsj1965.62.3_563.

Lamb, D., and W. D. Scott, 1974: The mechanism of ice crystal growth and habit formation. J. Atmos. Sci., 31, 570-580, https://doi.org/ 10.1175/1520-0469(1974)031<0570:TMOICG > 2.0.CO;2.

, and J. Verlinde, 2011: Physics and Chemistry of Clouds. Cambridge University Press, 584 pp.

Leinonen, J., S. Kneifel, D. Moisseev, J. Tyynelä, S. Tanelli, and T. Nousiainen, 2012: Evidence of nonspheroidal behavior in millimeter-wavelength radar observations of snowfall. J. Geophys. Res., 117, D18205, https://doi.org/10.1029/2012JD017680.

Libbrecht, K., 2003: Growth rates of the principal facets of ice between $-10^{\circ} \mathrm{C}$ and $-40^{\circ} \mathrm{C}$. J. Cryst. Growth, 247, 530-540, https://doi.org/10.1016/S0022-0248(02)01996-6.

Lin, Y. L., R. D. Farley, and H. D. Orville, 1983: Bulk parameterization of the snow field in a cloud model. J. Climate Appl. Meteor., 22, 1065-1092, https://doi.org/10.1175/1520-0450(1983) 022<1065:BPOTSF $>2$.0.CO;2.

Liou, K. N., 1976: On the absorption, reflection and transmission of solar radiation in cloudy atmospheres. J. Atmos. Sci., 33, 798-805, https:// doi.org/10.1175/1520-0469(1976)033<0798:OTARAT>2.0.CO;2. , 1986: Influence of cirrus clouds on weather and climate processes: A global perspective. Mon. Wea. Rev., 114, 1167-1199, https:// doi.org/10.1175/1520-0493(1986)114<1167:IOCCOW>2.0.CO;2. 
Liu, G., 2008: A database of microwave single-scattering properties for nonspherical ice particles. Bull. Amer. Meteor. Soc., 89, 1563-1570, https://doi.org/10.1175/2008BAMS2486.1.

Lord, S. J., H. E. Willoughby, and J. M. Piotrowicz, 1984: Role of a parameterized ice-phase microphysics in an axisymmetric, nonhydrostatic tropical cyclone model. J. Atmos. Sci., 41, 2836-2848, https://doi.org/10.1175/1520-0469(1984)041<2836: ROAPIP $>2.0 . C O ; 2$.

Lu, Y., Z. Jiang, K. Aydin, J. Verlinde, E. E. Clothiaux, and G. Botta, 2016: A polarimetric scattering database for non-spherical ice particles at microwave wavelengths. Atmos. Meas. Tech., 9, 5119-5134, https://doi.org/10.5194/amt-9-5119-2016.

Marshall, J. S., and M. P. Langleben, 1954: A theory of snow-crystal habit and growth. J. Meteor., 11, 104-120, https://doi.org/ 10.1175/1520-0469(1954)011<0104:ATOSCH>2.0.CO;2.

Matrosov, S. Y., R. F. Reinking, and I. V. Djalalova, 2005: Inferring fall attitudes of pristine dendritic crystals from polarimetric radar data. J. Atmos. Sci., 62, 241-250, https://doi.org/10.1175/JAS-3356.1.

Metcalf, J. I., 1988: A new slant on the distribution and measurement of hydrometeor canting angles. J. Atmos. Oceanic Technol., 5, 571-578, https://doi.org/10.1175/1520-0426(1988) 005<0571:ANSOTD>2.0.CO;2.

Mitchell, D. L., 1996: Use of mass- and area-dimensional power laws for determining precipitation particle terminal velocities. J. Atmos. Sci., 53, 1710-1723, https://doi.org/10.1175/15200469(1996)053<1710:UOMAAD > 2.0.CO;2.

, and A. J. Heymsfield, 2005: Refinements in the treatment of ice particle terminal velocities, highlighting aggregates. J. Atmos. Sci., 62, 1637-1644, https://doi.org/10.1175/JAS3413.1.

, R. Zhang, and R. L. Pitter, 1990: Mass-dimensional relationships for ice particles and the influence of riming on snowfall rates. J. Appl. Meteor., 29, 153-163, https://doi.org/ 10.1175/1520-0450(1990)029<0153:MDRFIP > 2.0.CO;2.

Mitra, S. K., O. Volhl, M. Ahr, and H. R. Pruppacher, 1990: A wind tunnel and theoretical study of the melting behavior of atmospheric ice particles. Part IV: Experiment and theory for snow flakes. J. Atmos. Sci., 47, 584-591, https://doi.org/ 10.1175/1520-0469(1990)047<0584:AWTATS>2.0.CO;2.

Morrison, H., and W. W. Grabowski, 2008: A novel approach for representing ice microphysics in models: Description and tests using a kinematic framework. J. Atmos. Sci., 65, 1528-1548, https://doi.org/10.1175/2007JAS2491.1.

Nadarajah, S., and S. Kotz, 2005: Some bivariate beta distributions. Statistics, 39, 457-466, https://doi.org/10.1080/ 02331880500286902.

Nelson, J., and C. Knight, 1998: Snow crystal habit changes explained by layer nucleation. J. Atmos. Sci, 55, 1452-1465, https://doi.org/ 10.1175/1520-0469(1998)055<1452:SCHCEB > 2.0.CO;2.

Nowell, H., G. Liu, and R. Honeyager, 2013: Modeling the microwave single-scattering properties of aggregate snowflakes. J. Geophys. Res. Atmos., 118, 7873-7885, https://doi.org/ 10.1002/jgrd.50620.

Ori, D., T. Maestri, R. Rizzi, D. Cimini, M. Montopoli, and F. S. Marzano, 2014: Scattering properties of modeled complex snowflakes and mixed-phase particles at microwave and millimeter frequencies. J. Geophys. Res. Atmos., 119, 9931-9947, https://doi.org/10.1002/2014JD021616.

Pruppacher, H. R., and J. D. Klett, 1997: Microphysics of Clouds and Precipitation: With an Introduction to Cloud Chemistry and Cloud Electricity. Kluwer Academic, 954 pp.

Ramanathan, V., and W. Collins, 1991: Thermodynamic regulation of ocean warming by cirrus clouds deduced from observations of the 1987 El Niño. Nature, 351, 27-32, https://doi.org/10.1038/351027a0.
- R. D. Cess, E. F. Harrison, P. Minnis, B. R. Barkstrom, E. Ahmad, and D. Hartmann, 1989: Cloud-radiative forcing and climate: Results from the Earth Radiation Budget Experiment. Science, 243, 57-63, https://doi.org/10.1126/ science.243.4887.57.

Rutledge, S. A., and P. V. Hobbs, 1984: The mesoscale and microscale structure and organization of clouds and precipitation in midlatitude cyclones. XII: A diagnostic modeling study of precipitation development in narrow cold-frontal rainbands. J. Atmos. Sci., 41, 2949-2972, https://doi.org/10.1175/15200469(1984)041<2949:TMAMSA > 2.0.CO;2.

Ryzhkov, A., M. Pinsky, A. Pokrovsky, and A. Khain, 2011: Polarimetric radar observation operator for a cloud model with spectral microphysics. J. Appl. Meteor. Climatol., 50, 873-894, https://doi.org/10.1175/2010JAMC2363.1.

Sassen, K., 1987: Polarization and Brewster angle properties of light pillars. J. Opt. Soc. Amer., 4A, 570-580, https://doi.org/ 10.1364/JOSAA.4.000570.

Sazaki, G., S. Zepeda, S. Nakatsubo, E. Yokoyama, and Y. Furukawa, 2010: Elementary steps at the surface of ice crystals visualized by advanced optical microscopy. Proc. Natl. Acad. Sci. USA, 107, 19702-19707, https://doi.org/10.1073/pnas.1008866107.

Schmitt, C. G., and A. J. Heymsfield, 2010: The dimensional characteristics of ice crystal aggregates from fractal geometry. J. Atmos. Sci., 67, 1605-1616, https://doi.org/10.1175/2009JAS3187.1.

$\longrightarrow,-$, P. Connolly, E. Järvinen, and M. Schnaiter, 2016: A global view of atmospheric ice particle complexity. Geophys. Res. Lett., 43, 11913-11 920, https://doi.org/10.1002/2016GL071267.

Sieron, S. B., F. Zhang, E. E. Clothiaux, L. N. Zhang, and Y. Lu, 2018: Representing precipitation ice species with both spherical and nonspherical particles for radiative transfer modeling of microphysics-consistent cloud microwave scattering properties. J. Adv. Model. Earth Syst., 10, 1011-1028, https:// doi.org/10.1002/2017MS001226.

Thériault, J. M., R. Rasmussen, K. Ikeda, and S. Landolt, 2012: Dependence of snow gauge collection efficiency on snowflake characteristics. J. Appl. Meteor. Climatol., 51, 745-762, https:// doi.org/10.1175/JAMC-D-11-0116.1.

Thompson, G., P. R. Field, R. M. Rasmussen, and W. D. Hall, 2008: Explicit forecasts of winter precipitation using an improved bulk microphysics scheme. Part II: Implementation of a new snow parameterization. Mon. Wea. Rev., 136, 5095-5115, https://doi.org/10.1175/2008MWR2387.1.

Tyynelä, J., and V. Chandrasekar, 2014: Characterizing falling snow using multifrequency dual-polarization measurements. J. Geophys. Res. Atmos., 119, 8268-8283, https://doi.org/10.1002/2013JD021369.

Vavrus, S., 2004: The impact of cloud feedbacks on Arctic climate under greenhouse forcing. J. Climate, 17, 603-615, https:// doi.org/10.1175/1520-0442(2004)017<0603:TIOCFO > 2.0.CO;2.

Voth, G. A., and A. Soldati, 2017: Anisotropic particles in turbulence. Annu. Rev. Fluid Mech., 49, 249-276, https://doi.org/ 10.1146/annurev-fluid-010816-060135.

Waliser, D. E., and Coauthors, 2009: Cloud ice: A climate model challenge with signs and expectations of progress. J. Geophys. Res., 114, D00A21, https://doi.org/10.1029/2008JD010015.

Westbrook, C. D., R. C. Ball, P. R. Field, and A. J. Heymsfield, 2004: Universality in snowflake aggregation. Geophys. Res. Lett., 31, L15104, https://doi.org/10.1029/2004GL020363.

Wu, W., and G. M. McFarquhar, 2016: On the impacts of different definitions of maximum dimension for nonspherical particles recorded by $2 \mathrm{D}$ imaging probes. J. Atmos. Oceanic Technol., 33, 1057-1072, https://doi.org/10.1175/JTECHD-15-0177.1. 